This is the final peer-reviewed accepted manuscript of:

Delbono, Flavio, and Luca Lambertini. "On the properties of linear supply functions in oligopoly." Economics Letters 136 (2015): 22-24.

The final published version is available online at:

https://doi.org/10.1016/j.econlet.2015.08.027

(C) 2015 This manuscript version is made available under the Creative Commons AttributionNonCommercial-NoDerivs (CC BY-NC-ND) 4.0 International License

(http://creativecommons.org/licenses/by-nc-nd/4.0/) 


\title{
On the properties of linear supply functions in oligopoly*
}

\author{
Flavio Delbono ${ }^{\#}$ and Luca Lambertini ${ }^{\S}$ \\ \# Department of Economics, University of Bologna \\ Piazza Scaravilli 2, 40126 Bologna, Italy \\ flavio.delbono@unibo.it \\ $\S$ Department of Economics, University of Bologna \\ Strada Maggiore 45, 40125 Bologna, Italy \\ luca.lambertini@unibo.it
}

August 17, 2015

\begin{abstract}
In this note we revisit the result by Menezes and Quiggin (2012), showing that under linear supply function competition, the same Nash equilibrium results when firms choose slopes or intercepts of their supply functions. This is because the first order conditions emerging in the two strategy spaces are not linearly independent.
\end{abstract}

JEL Codes: D43, L13

Keywords: supply function; linear independence

*We thank an anonymous referee for helpful suggestions. The usual disclaimer applies. 


\section{Introduction}

After the seminal paper by Klemperer and Meyer (1989), the literature on supply functions has been growing in several directions. This is no surprise, because supply function competition represents an interesting option when modelling oligopolistic interaction. However, the complexity of the Klemperer and Meyer (1989) setting has stimulated approaches designed to make their original model more tractable.

More precisely, we have witnessed a special focus on linear supply functions. One of the models proposed in this vein is in Menezes and Quiggin (2012), where firms choose the intercept of their linear supply functions, while the slope is treated as a parameter, in such a way that, if the slope is nil, the equilibrium outcome replicates Cournot, whereas if the slope is infinitely high, it replicates Bertrand or perfect competition.

Menezes and Quiggin's (2012) formulation, however, allows one to treat also the slope as a strategic variable. This is what we do in this paper. We show that the resulting first order conditions in the extended strategy space formed by both intercepts and slopes are not linearly independent and the Nash equilibrium generated by choosing either one is the same.

We set up the model in section 2. Section 3 contains our results, and section 4 concludes by underlying some extensions.

\section{The model}

Consider an oligopoly formed by $n$ identical firms selling a homogeneous good whose market demand function is $p=a-Q$, where $p$ is market price, $a>0$ is a parameter measuring market size (which is normalised to one in 
Menezes and Quiggin (2012)) and industry output $Q=\sum_{i=1}^{n} q_{i}$ is the sum of all individual outputs $q_{i}$. Firm $i$ 's cost function is $C_{i}=c q_{i}$ where parameter $c \in(0, a)$. Menezes and Quiggin (2012) specify the individual firm's supply function as $q_{i}=\alpha_{i}-c / n+\beta(p-c)$, where intercept $\alpha_{i}>0$ is the strategic variable, while slope $\beta \geq 0$ is a parameter. It is worth noting that this supply function is increasing in the mark-up; the intercept is normalised the characterisation of equilibrium.

In view of Ciarreta and Gutierrez-Hita (2006), where the supply function is $q_{i}=\beta_{i} p$ and the slope is the strategic variable, we extend Menezes and Quiggin's (2012) approach by stipulating that the supply function is as follows:

$$
q_{i}=\alpha_{i}-\frac{c}{n}+\beta_{i}(p-c)
$$

where both the intercept $\alpha_{i}>0$ and the slope $\beta_{i} \geq 0$ are strategic variables.

Solving the ex ante market-clearing condition, we get

$$
p=\frac{a-\sum_{i=1}^{n} \alpha_{i}+c\left(1+\sum_{i=1}^{n} \beta_{i}\right)}{1+\sum_{i=1}^{n} \beta_{i}}
$$

so that the individual profit function is

$$
\begin{gathered}
\pi_{i}=(p-c) q_{i}= \\
{\left[\frac{a-\sum_{i=1}^{n} \alpha_{i}+c\left(1+\sum_{i=1}^{n} \beta_{i}\right)}{1+\sum_{i=1}^{n} \beta_{i}}-c\right]\left(\alpha_{i}-\frac{c}{n}\right)+} \\
\beta_{i}\left[\frac{a-\sum_{i=1}^{n} \alpha_{i}+c\left(1+\sum_{i=1}^{n} \beta_{i}\right)}{1+\sum_{i=1}^{n} \beta_{i}}-c\right]^{2}
\end{gathered}
$$

\section{Results}

While Menezes and Quiggin (2012, p. 713) assume $\beta_{i}=\beta$ for all $i=$ $1,2,3, \ldots, n$ and take first order conditions (FOCs) w.r.t. $\alpha_{i}$ 's, we calculate 
the FOCs w.r.t. both $\alpha_{i}$ 's and $\beta_{i}$ 's:

$$
\begin{gathered}
\frac{\partial \pi_{i}}{\partial \alpha_{i}}=\frac{c \Phi-n\left[\left(2 \alpha_{i}+\sum_{j \neq i} \alpha_{j}\right) \Theta+a\left(\beta_{i}-\Theta\right)\right]}{n \Phi^{2}}=0 \\
\frac{\partial \pi_{i}}{\partial \beta_{i}}=\frac{\left(a-\sum_{i=1}^{n} \alpha_{i}\right)\left[c \Phi-n\left(\left(2 \alpha_{i}+\sum_{j \neq i} \alpha_{j}\right) \Theta+a\left(\beta_{i}-\Theta\right)\right)\right]}{n \Phi^{3}}=0
\end{gathered}
$$

where $\Phi \equiv 1+\sum_{i=1}^{n} \beta_{i}$ and $\Theta \equiv 1+\sum_{j \neq i} \beta_{j}$. Looking at the system (4-5), it appears that

$$
\frac{\partial \pi_{i}}{\partial \beta_{i}}=\frac{\partial \pi_{i}}{\partial \alpha_{i}} \cdot \frac{a-\sum_{i=1}^{n} \alpha_{i}}{\Phi}
$$

which implies the following:

Lemma 1 If the strategy space of the game in supply functions includes both the slope and the intercept of each firm's supply, strategic variables are not linearly independent.

The above Lemma immediately implies a relevant consequence:

Proposition 2 Under linear supply competition, the Nash equilibrium is independent of the combination of intercepts and slopes chosen by firms.

That is to say, the Nash equilibrium of this game can be equivalently obtained by (i) posing $\beta_{i}=\beta$ in (4) and then solving for $\alpha_{i}$ or (ii) posing $\alpha_{i}=\alpha$ in (5) and then solving for $\beta_{i}$. This entails that firm $i$ may not even know (and in fact, doesn't care to know) whether any rival firm $j$ is using (4) or (5).

It is a matter of simple algebra to show that the two methods indeed lead to the same outcome. Procedure (i) yields the same Nash equilibrium solution as in Menezes and Quiggin (2012, eq. (5), p. 713):

$$
\alpha^{*}=\frac{c(1+n \beta)+a n[1+\beta(n-2)]}{n[n+1+n \beta(n-1)]}
$$


Procedure (ii) yields

$$
\beta^{*}=\frac{c+n[a-\alpha(n+1)]}{n[n \alpha(n-1)-a(n-2)-c]}
$$

and then it is easily verified that $\alpha^{*}$ and $\beta^{*}$ are invertible, with $\alpha^{*}=\left(\beta^{*}\right)^{-1}$. Using $\alpha^{*}$, the equilibrium price $p^{*}$ is as in Menezes and Quiggin (2012, eq. (6), p. 173), with

$$
\left.p^{*}\right|_{\beta=0}=\frac{a+c n}{n+1} ; \lim _{\beta \rightarrow \infty} p^{*}=c
$$

reproducing the special cases of Cournot and Bertrand (or perfect competition), respectively. If one uses instead $\beta^{*}$, the resulting price is

$$
p^{*}=\frac{c+n \alpha(n-1)-a(n-2)}{2}
$$

with

$$
p^{*}=\frac{a+c n}{n+1} \text { at } \alpha=\frac{a n+c}{n(n+1)}=\left.\alpha^{*}\right|_{\beta=0}
$$

and

$$
p^{*}=c \text { at } \alpha=\frac{a(n-2)+c}{n(n-1)}=\lim _{\beta \rightarrow \infty} \alpha^{*}
$$

Then, the equivalence between the two above procedures is complete.

The intuition behind this result can be explained as follows. Under the market clearing condition, each firm operates along the residual demand curve left by the supply chosen by rivals. Hence, since supply functions are linear, it's indifferent to determine the optimal supply by choosing the intercept or the slope. To see this, take the standpoint of firm $i$, whose supply function is defined in (1), and suppose firm $i$ takes the rivals' collective supply $Q_{-i}=\sum_{j \neq i} q_{j}$ as given. Hence, the residual demand faced by firm $i$ is $D_{R}=a-p-Q_{-i}$, which, by the market clearing condition, must be equal to $q_{i}$. Imposing $Q_{-i}=(n-1) q_{j}$ and solving $D_{R}=q_{i}$ w.r.t. price, we obtain

$$
p=\frac{n\left[a-\alpha_{i}-(n-1) q_{j}\right]+c\left(n \beta_{i}+1\right)}{n\left(1+\beta_{i}\right)}
$$


Firm $i$ 's profits are again defined as $\pi_{i}=(p-c) q_{i}$. Taking FOCs w.r.t. $\alpha_{i}$ and $\beta_{i}$, we get

$$
\begin{gathered}
\frac{\partial \pi_{i}}{\partial \alpha_{i}}=\frac{n\left[a\left(1-\beta_{i}\right)-2 \alpha_{i}+\left(\beta_{i}-1\right)(n-1) q_{j}\right]+c\left[2+n\left(\beta_{i}-1\right)\right]}{n\left(1+\beta_{i}\right)^{2}}=0 \\
\frac{\partial \pi_{i}}{\partial \beta_{i}}=-\frac{c(n-1)+n\left[\alpha_{i}-a+(n-1) q_{j}\right]}{n\left(1+\beta_{i}\right)} \cdot \frac{\partial \pi_{i}}{\partial \alpha_{i}}=0
\end{gathered}
$$

which jointly imply that the above intuitive explanation is indeed correct.

\section{Extensions}

The result stated in Proposition 1 extends to a more general setup in which the cost function includes a quadratic component, becoming $C_{i}=q_{i}\left(c+b q_{i} / 2\right)$, as in Ciarreta and Gutierrez-Hita (2006). ${ }^{1}$ In this case, one may simply rewrite the individual supply function as

$$
q_{i}=\alpha_{i}-\frac{\partial C_{i} / \partial q_{i}}{n}+\beta_{i}(p-c)
$$

and then proceed as follows. Taking FOCs w.r.t. $\alpha_{i}$ and $\beta_{i}$ and imposing the symmetry conditions $\alpha_{j}=\alpha_{i}$ and $\beta_{j}=\beta_{i}$ for all $j \neq i$, we obtain the following expressions:

$$
\begin{aligned}
& \frac{\partial \pi_{i}}{\partial \alpha_{i}}=\frac{n}{(b+n)\left[b+n\left(n \beta_{i}+1\right)\right]^{2}}\left[(b+n)\left(c+a(b+n)-n(n+1+b) \alpha_{i}\right)-\right. \\
& \left.n(b+n)\left(n(n-1) \alpha_{i}-(1+b) c-a(n-2-b)\right) \beta_{i}-b(a-c)(n-1) n^{2} \beta_{i}^{2}\right]=0 \\
& \frac{\partial \pi_{i}}{\partial \beta_{i}}=-\frac{n\left[a(b+n)-b c-n^{2} \alpha_{i}\right]}{(b+n)\left[b+n\left(n \beta_{i}+1\right)\right]^{3}}\left[(b+n)\left(c+a(b+n)-n(n+1+b) \alpha_{i}\right)-\right.
\end{aligned}
$$

\footnotetext{
${ }^{1}$ In Klemperer and Meyer (1989), the marginal cost is actually assumed to be nonnegative.
} 
$\left.n(b+n)\left(n(n-1) \alpha_{i}-(1+b) c-a(n-2-b)\right) \beta_{i}-b(a-c)(n-1) n^{2} \beta_{i}^{2}\right]=0$

so that we may write

$$
\frac{\partial \pi_{i}}{\partial \beta_{i}}=-\frac{\partial \pi_{i}}{\partial \alpha_{i}} \cdot \frac{a(b+n)-b c-n^{2} \alpha_{i}}{\left[b+n\left(n \beta_{i}+1\right)\right]}
$$

which proves that (17) and (18) are not linearly independent. Consequently, it can also be shown that exactly the same equilibrium outcome arises if the game takes a two-stage structure in which the first stage takes place in the $\alpha$-space and the second stage in the $\beta$-space (or the opposite).

Finally, one may wonder about the specific normalisation adopted by Menezes and Quiggin (2012). It can be easily shown that our result does not depend on it, by reconstructing the foregoing argument under the assumption that the supply function is written as $q_{i}=\alpha_{i}+\beta_{i} p$.

\section{References}

[1] Ciarreta, A. and C. Gutierrez-Hita (2006), "Supply Function vs Quantity Competition in Supergames", International Journal of Industrial Organization, 24, 773-83.

[2] Klemperer, P.D. and M.A. Meyer (1989), "Supply Function Equilibria in Oligopoly under Uncertainty", Econometrica, 57, 1243-77.

[3] Menezes, F.M. and J. Quiggin (2012), "More Competitors or More Competition? Market Concentration and the Intensity of Competition", Economics Letters, 117, 712-14. 